\title{
The Role of Smart Personal Assistant for improving personal Healthcare
}

\author{
Arul Srinivasan, A.Neela Madheswari \\ CSE Department, Mahendra Engineering College, Namakkal, India \\ Email: neela.madheswari@gmail.com
}

\begin{abstract}
Machine learning is a subfield of computer science that evolved from the study of pattern recognition and computational learning theory in artificial intelligence. The focus of this paper is to improve personal healthcare using smart personal assistant which make use of the combination of machine learning and cloud. Making a doctor appointment through phone call is a tedious process and it may take more time. People who did not made the prior appointment have to wait on the queue which sometimes leads to dissatisfactions to the patients. To overcome this gap, smart personal assistant application is proposed using which users can get appointment of various doctors according to the current availability and at anytime and anywhere. This will improve time saving from patients' side as well as they will be satisfied by timely service.
\end{abstract}

Keywords - cloud service, healthcare, machine learning, smart personal assistant

\section{INTRODUCTION}

Time plays a major role in everyone's life. We are in the digital era. We never want to wait to receive or buy anything if it takes more time. If the waiting time for obtaining any goods or products means we will be looking for a better any other option or chance so that we can receive our goods or products in a timely manner. Smart personal assistants (SPA) play a major role nowadays. People are now moving towards SPAs. The implementation of context-aware computing is possible via personal assistants known as User Assistance Systems, virtual agents or SPAs, such as Google Assistant, Amazon Alexa, Microsoft Cortana or Samsung Bixby [1]. There are considerable differences between them.

Maedche et al. suggested a classification based on two dimensions: i) the degree of intelligence of the system, ii) the degree of interaction implemented by the system. In contrast to basic systems characterized by a low degree of interaction and low degree of intelligence, advanced systems are characterized by more sophisticated features. They allow users to decide whether to follow the assistance, provide a high extent of context-aware and proactive assistance, include adaptation capabilities, and detect users' needs [2].

Digital personal assistants in the form of chatbots offer a lot more than simple messaging apps. They can be voice controlled, which makes it possible to use them also when our hands are full with any other activities [3].

Nowadays people are more connected and in healthcare organizations also use new innovative technologies to improve patient care. Voice assistants and voice enabled devices also offer significant promise to increase patient engagement, improve outcomes and reduce cost. Most of the healthcare institutions are already using voice enabled intelligent devices to improve patients' healthcare.

\section{RELATED WORK}

A personal digital assistant or handheld computer is a small, mobile, handheld device that provides computing and information storage/retrieval capabilities. Recent years have seen a significant increase in the deployment of voice-controlled personal digital assistants. Some of the example products are Apple's Siri, Google's Now and Nuance's Nina. Some of the improvements possible are: i) extended dialog history, ii) improved context awareness, iii) dynamic system adaptation, and, iv) supported task hierarchy design [4].

The use of speech recognition is increasing rapidly and is now available in smart TVs, desktop computers, every new smart phones, etc allowing us to talk to computers naturally. With the use in home appliances, education and even in surgical procedures accuracy and speed becomes very important [5].

The growth in Human-Computer interaction field has not only been in quality of interaction, it has also experienced different branching in its history. Instead of designing regular interfaces, the different research branches had different focus on the concepts of multimodality, intelligent adaptive interfaces rather than command or action based, and finally active rather than passive interfaces [6].

PDAs are used in various fields. A study [7] described the usage of PDAs among medical students are analysed. The 
table below gives the application of PDAs for various factors by the students.

Table.1: Common uses of PDAs among medical students $(n=53)[7]$

\begin{tabular}{|l|l|l|}
\hline S.No & Purpose & $\begin{array}{l}\text { Number of } \\
\text { students }\end{array}$ \\
\hline 1 & Calendar & 48 \\
\hline 2 & Use drug reference & 41 \\
\hline 3 & Email/text & 26 \\
\hline 4 & Clinical reference text & 26 \\
\hline 5 & Dictionary & 22 \\
\hline 6 & Perform clinical calculations & 15 \\
\hline 7 & Access different diagnoses & 13 \\
\hline 8 & Take notes & 11 \\
\hline 9 & Access course information & 10 \\
\hline 10 & Take pictures & 9 \\
\hline 11 & Search for literature & 3 \\
\hline
\end{tabular}

A systematic review of surveys demonstrated that a PDA was more likely to be accepted and used among physicians and younger and those who were working in large and hospital-based practices. Although PDAs could not store or organize large graphics and patients' entire medical records, they have played a significant role in managing certain amount of electronic documentation and accessing it at the point of care easily. Using mobile technologies such as smart phones and tablets with high quality design investigating the effectiveness and efficiency of using mobile devices for specific tasks are needed [8].

\section{SYSTEM ARCHITECTURE}

The objective of this work is to build an AI personal assistant that helps user to schedule doctor appointment to a specific doctor at any time and place. The users have to find easily the nearby hospitals and availability status of the doctor. If the user wishes to schedule the appointment they have to pick the time slot and instant confirmation have to be made. In case if the doctor cancelled the appointment, the user can pick any other doctor appointment time slot. Rescheduling of the appointment also provided to give more comfortable to the user. In addition to the appointment scheduling system this application keeps track of the health record with the ML program it keeps on notify the user regarding the timely visit to the doctor and also help them to take their medicine on time in daily basis. It includes speech recognition system which will be easier to the user to communicate with it. It supports major languages being spoken in India so that even the normal people can easily interact without any conflicts. This application can be integrated in Facebook messenger, Skype, Telegram,
Website, Kik and slack community. This application is also available for android and windows platform. This application is need not to be installed by the user. All Android 5.0+ devices is preinstalled with Google Action App. This personal assistant can be triggered by launching the Google Action and just saying the app name. The Google Action will get connected to our personal assistant and can be executed there. This is easy for people and do not having the problem of downloading the application and also space issue in their device.

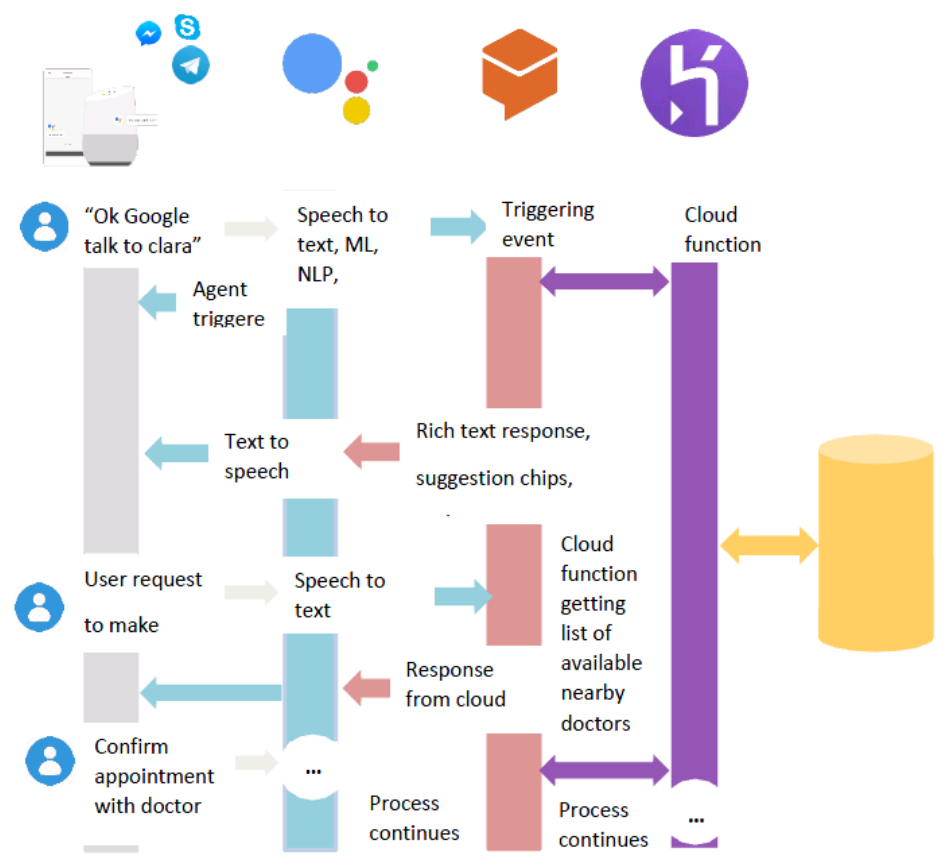

Fig. 1: System architecture

The dependencies used in this work to achieve our objective are given as follows:

1. Google Cloud Platform

2. Dialogflow

3. Heroku Cloud App Hosting

1. Google Cloud Platform:

In this work, the features of NLP (Natural Language Processing), ML (Machine Learning) and APIs (Application programming interface) are used from Google Cloud Platform. NL API is a REST (Representational State Transfer) and consists of JSON requests and response. All requests from cloud are given in the form of JSON. ML part is used to train the application for voice recognition. APIs are used to generate user interface while interacting with the application. 


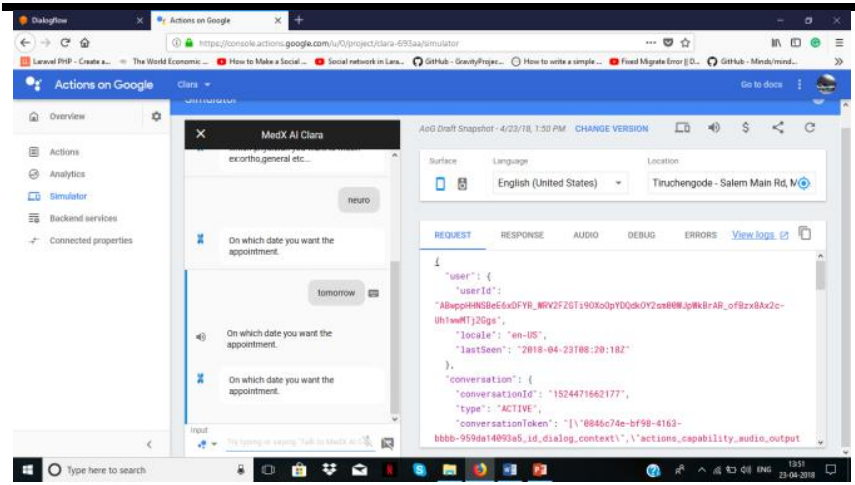

Fig. 2: Retrieving Cloud data (request in JSON format)

2. DialogFlow:

The main important functions to be involved using Dialogflow is the support of intent flow, platform integration and training the agent. To train the agent, Intent is used. Intents are used to map user input to responses. The Dialogflow has three main steps namely: i) User giving input, ii) Dialogflow agent parsing that input and iii) Agent returning a response to the user.

3. Heroku Cloud App Hosting:

To perform cloud functions, database access and backend code deployment, Heroku is used. Heroku is a cloud platform as a service supporting several programming languages.

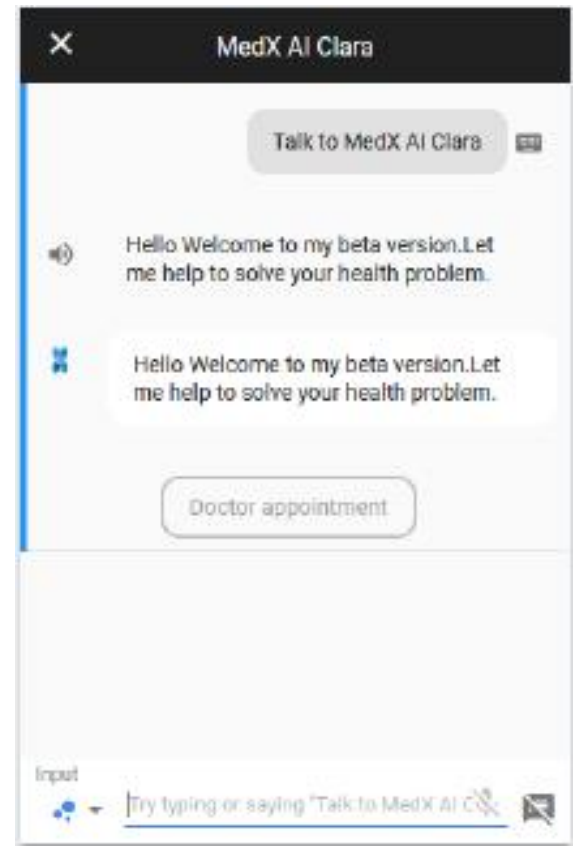

Fig. 3: MedX AI Clara interface

\section{SYSTEM ANALYSIS}

MedX AI Clara is the name of the application created. It is hosted and executed in cloud. First the interface of MedX AI Clara is shown in figure 3 below. The next selection is finding the doctor appointment. It is given in figure 4 . Then we have to select which category of doctor we want to see i.e. neuro specialist or dentist, etc. After giving proper category, we can get the details of the particular doctor and then we can fix the appointment using that interface itself.

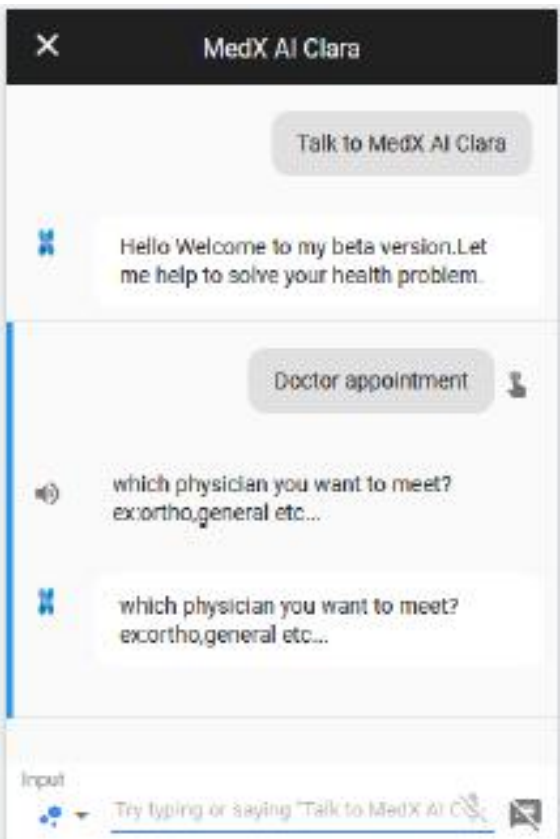

Fig. 4: Doctor appointment using MedX AI Clara
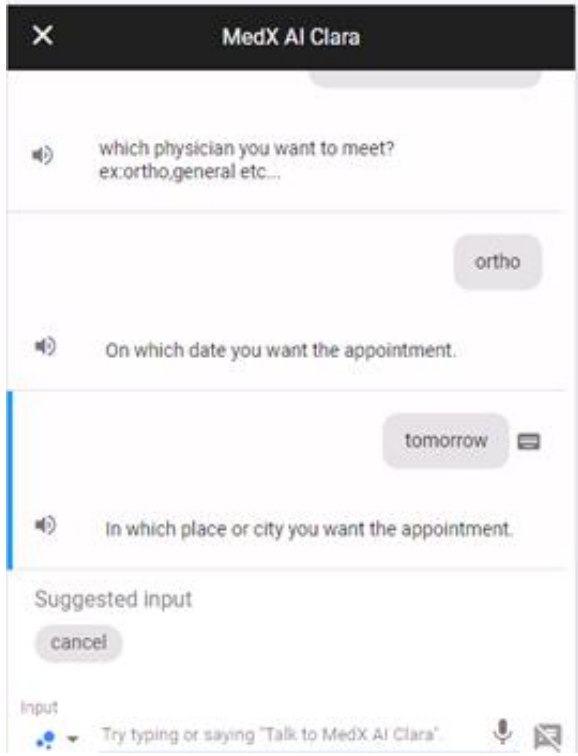

Fig. 5: Department selection \& date using MedX AI Clara 


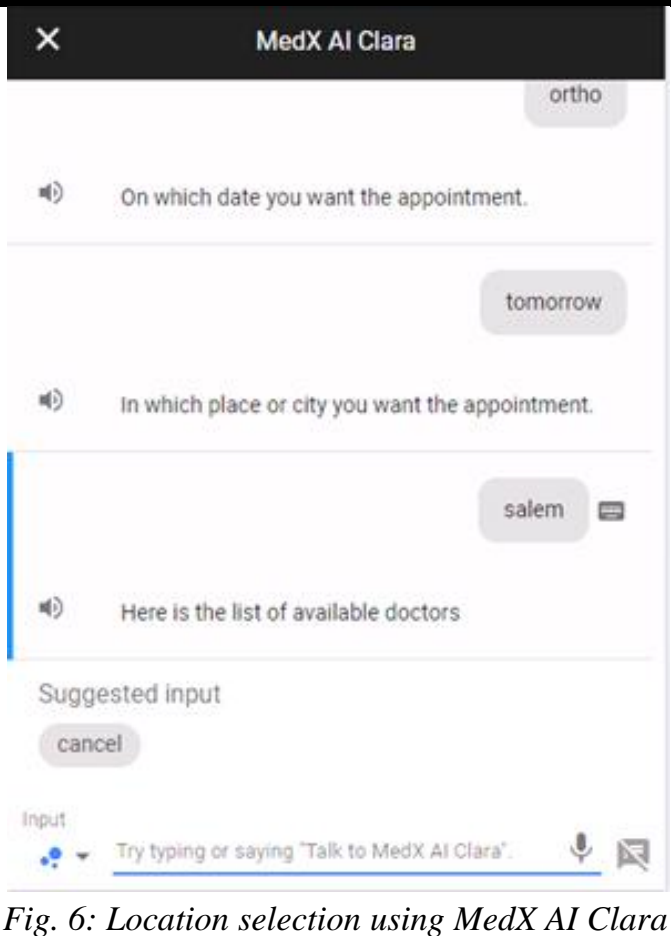

\section{RESPONSE}

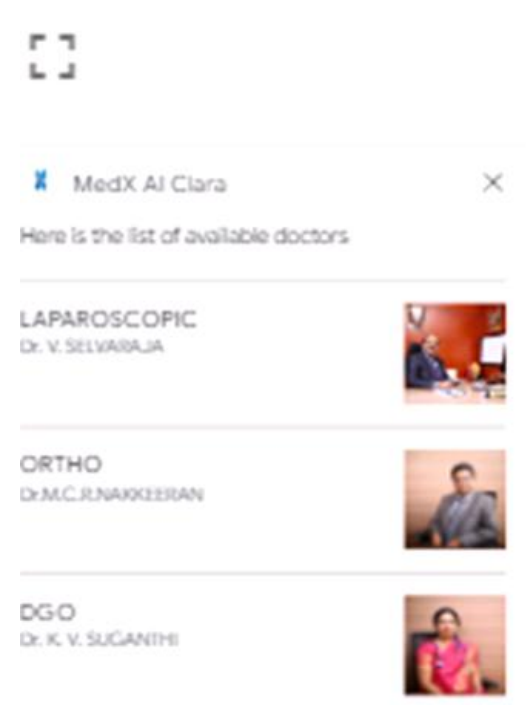

Fig. 7: Final response of available doctors using MedX AI Clara

\section{CONCLUSION}

The proposed system provides quick confirmation of appointment. it also provides easy interface and user can book appointment at any time with the doctor and at anywhere. another advantage of using this system is people can book appointment in a short time and it also avail voice recognition feature to increase the speed of execution. Since everything is hosted in Cloud environment, the users need not to install the developed application in his/her smart device since everything is hosted in cloud itself. If the device is capable of accessing Internet, then we can use this application anywhere which is smart enough since it reduces the time of interfacing for getting doctors appointment which is a time consuming process in traditional system practice. Also health is very essential factor and we have to get solution for the availability of doctors or specialists in emergency situation where we don't have enough time to talk and find the location and for appointment of those persons. This system will helpful for people who have smart devices and who are in need of smart solution for their healthcare.

\section{REFERENCES}

[1] Knote, R.; Janson, A.; Eigenbrod, L. \& Söllner, M. (2018): The What and How of Smart Personal Assistants: Principles and Application Domains for IS Reserach. In: Multikonferenz Wirtschaftsinformatik (MKWI). Lüneburg, Germany.

[2] Maedche. A, Morana S, Schacht S, Werth D, Knumeich J, Advanced User Assistance Systems, In: Business and Information Systems Engineering 58, 367-370 (2016).

[3] TMF, The Medical Futurist, "Chatbots will serve as Health assistants", April 2017, https://medicalfuturist.com/chatbots-health-assistants.

[4] P.Milhorat, S.Schlogl, G.Chollet, J.Boudy, A.Esposito, G.Pelosi, Building the Next generation of Personal digital Assistants, In: International Conference on Advanced Technologies for Signal and Image Processing (ATSIP), 2014.

[5] Magnus Stenman, Automatic Speech Recognition An Evaluation of Google Speech, UMEA Universitet, Spring 2015.

[6] Fakhreddine Karray, Milad Alemzadeh, Jamil Abou Saleh and Mo Nours Arab, "Human-Computer interaction:Overview on State of the Art", International Journal on Smart sensing and Intelligent systems, vol.1, no.1, March 2008.

[7] M. Jaiswal, "Cloud computing and Infrastructure", International Journal of Research and Analytical Reviews, vol. 4, no. 2, pp. 742-746, 2017.

[8] Trish Chatterley, Dagmara Chojecki, "Personal Digital assistant usage among undergraduate medical students: Exploring trends, barriers, and the advent of smartphones, Journal of the Medical Library Association, April 2010.

[9] Ping Guo, Kim Watts, Heather Wharrad, "An integrated review of the impact of mobile technologies used by healthcare professionals to support education and practice", Nursing Open, Sep 2015 . 